\title{
HIPOGLUCEMIA HIPERINSULINÉMICA POSTPRANDIAL LUEGO DE LA CIRUGÍA BARIÁTRICA
}

\author{
POSTPRANDIAL HYPERINSULINEMIC HYPOGLYCEMIA \\ AFTER BARIATRIC SURGERY
}

\author{
María Graciela Álvarez¹, Susana Gutt², María Yuma³, Paola Polo4, Sandra González5, Estrella Menéndez \\ Guadalupe Vanoli ${ }^{7}$, Hugo Montemerlo ${ }^{8}$, Marianela Ackerman ${ }^{9}$, Jimena Coronel ${ }^{10}$, Carla Gauna ${ }^{11}$, \\ Victoria Salinas ${ }^{12}$, Carla Musso ${ }^{13}$, Juliana Mociulsky ${ }^{14}$, Susana Fuentes ${ }^{15}$
}

\section{RESUMEN}

La incidencia de complicaciones informadas luego de la cirugía bariátrica (CB) ha ido en aumento en relación con la epidemia de obesidad y el mayor número de procedimientos quirúrgicos realizados a nivel mundial. Entre las complicaciones, la hipoglucemia hiperinsulinémica postprandial (HHP) adquirió relevancia, aunque es una de las menos conocidas y comprendidas de la $\mathrm{CB}$, frecuentemente inadvertida y por lo tanto subdiagnosticada. Se distingue de la hipoglucemia de ayuno en que su presentación es típicamente posterior a la ingesta, asociada a valores de hipoglucemia precedidos por hiperglucemia e hiperinsulinemia en sangre. Se caracteriza por el incremento de la variabilidad de la glucemia, con absorción acelerada, aumento y rápida caída del azúcar en sangre, por lo que comprende valores de hiper e hipoglucemia. Debe sospecharse cuando se detectan síntomas adrenérgicos o de neuroglucopenia postprandiales luego de la CB'. Ha sido considerada más frecuente luego del bypass gástrico en-Y-Roux (BGYR), pero también fue descripta asociada a otras técnicas quirúrgicas, como la derivación biliopancreática/ switch duodenal (DBP/SD) ${ }^{2}$ y la gastrectomía vertical en manga (GVM), y de otras intervenciones que comprometen el píloro o el vaciamiento gástrico ${ }^{3}$. No se observó luego de la cirugía con banda gástrica ajustable, un procedimiento que no altera la anatomía gastrointestinal ${ }^{4-5}$.

Palabras clave: hipoglucemia hiperinsulinémica; hipoglucemia postprandial; cirugía bariátrica; bypass gástrico; dumping tardío.

Revista de la Sociedad Argentina de Diabetes 2019; Vol. 53 (16-27)

\section{ABSTRACT}

Associated with the obesity epidemic and the growing number of bariatric surgery procedures being performed worldwide, the incidence of reported complications has increased as well. Among these, the postprandial hyperinsulinemic hypoglycemia (PHH) warrants further attention. This condition is not entirelly understood and is probably underdiagnosed. The PHH is characterized by hypoglycemic symptoms occurring after a meal accompanied by a low plasma glucose value, typically preceded by a high rise in both glucose and insulin concentrations. Patients with $\mathrm{PHH}$ have shown increased glucose variability with a rapid increase in glucose absorption into the systemic circulation and an increase in glucose disappearance, with glucose values reaching both the hyperglycemic and hypoglycemic areas. It should be suspected in patients with postprandial adrenergic or neuroglycopenic symptoms after bariatric surgery?.

It was considered most commonly associated with Roux-en Y gastric bypass (RYGB), although it was also described after biliopancreatic diversion (BPD) with duodenal switch ${ }^{2}$ and sleeve gastrectomy, and other surgical procedures which compromises the pylorus and the gastric emptying ${ }^{3}$. It has not been observed after adjustable gastric banding, an intervention that does not alter the gastrointestinal anatomy ${ }^{4-5}$.

Key words: hyperinsulinemic hypoglycemia; postprandial hypoglycemia; bariatric surgery; gastric bypass; late dumping.

Revista de la Sociedad Argentina de Diabetes 2019; Vol. 53 (16-27)
Médica especialista en Nutrición, Coordinadora del Centro de Estudio y Tratamiento de la Obesidad Severa (CETOS), Ciudad Autónoma de Buenos Aires, Argentina

2 Médica especialista en Nutrición, Jefa de la Sección Nutrición, Servicio de Clínica Médica, Hospital Italiano de Buenos Aires, Ciudad Autónoma de Buenos Aires, Argentina

3 Médica especialista en Nutrición, Magister en Diabetes, Médica de Planta, Sección Nutrición, Servicio de Clínica Médica, Hospital Italiano de Buenos Aires, Ciudad Autónoma de Buenos Aires, Argentina

4 Médica Endocrinóloga, Sanatorio Las Lomas, Médica de Planta del Servicio de Endocrinología, Hospital San José de la Ciudad de Campana, Provincia de Buenos Aires, Argentina
5 Médica del Consultorio de Diabetes, Clínica IMA, Adrogué, Provincia de Buenos Aires, Argentina

6 Médica especialista en Nutrición, Médica de Planta del Servicio de Nutrición y Diabetes, CEMIC, Ciudad Autónoma de Buenos Aires, Argentina

7 Médica especialista en Nutrición, Médica de Planta de la Unidad de Nutrición y Diabetes del Hospital General de Agudos José María Ramos Mejía, Provincia de Buenos Aires, Argentina

8 Médico especialista en Medicina Interna y Nutrición, Sanatorio Mater Dei, Ciudad Autónoma de Buenos Aires, Argentina

9 Médica especialista en Medicina Interna y Nutrición, Magister en Diabetes, Centro CIEN, Corrientes, Argentina 
10 Médica especialista en Medicina Interna, Centro Médico Maffei, Ciudad Autónoma de Buenos Aires, Argentina

11 Médica especialista en Clínica Médica y Nutrición, Medical Scientific Liason Diabetes y Obesidad, Novo Nordisk, Ciudad Autónoma de Buenos Aires, Argentina

12 Médica especialista en Clínica y Nutrición, integrante del Departamento de Clínica Médica y Nutrición del CEMIC, Ciudad Autónoma de Buenos Aires, Argentina

13 Médica Endocrinóloga, Coordinadora de Diabetes, Fundación Favaloro, Médica de Planta de la Unidad Asistencial César Milstein, PAMI, Ciudad Autónoma de Buenos Aires, Argentina
14 Médica Endocrinóloga, Jefa de la Unidad Nutrición, Instituto Cardiovascular Buenos Aires (ICBA), Ciudad Autónoma de Buenos Aires, Argentina

15 Médica especialista en Medicina Interna, Médica del Hospital El Cruce S.A.M.I.C, Provincia de Buenos Aires, Argentina

Contacto de la autora: María Graciela Álvarez

E-mail: mgalvarez@intramed.net

Correspondencia: Santa Fe 3996 7 81, (C1425BHO), Ciudad Autónoma de Buenos Aires, Argentina

Fecha de trabajo recibido: 05/11/18

Fecha de trabajo aceptado: 03/04/19

Conflictos de interés: los autores declaran que no existe conflicto de interés.

\section{INTRODUCCIÓN}

La hipoglucemia postprandial se describió en el año 1940 como una complicación de la resección gástrica en pacientes con úlcera péptica ${ }^{6}$. En el año 2005 se refirieron seis pacientes con hipoglucemia hiperinsulinémica postprandial (HHP) luego de BGYR asociadas a la presencia de nesidioblastosis, una entidad rara y de muy baja prevalencia?. Se consideró entonces que la HHP podía atribuirse a la hiperplasia de las células $\beta$ de los islotes pancreáticos. Posteriormente esos datos se revisaron y cuestionaron; desde entonces el debate creció y se establecieron varias hipótesis sobre su fisiopatología.

Actualmente la HHP se adjudica a los cambios anatómicos resultantes de la cirugía bariátrica (CB) que alteran la cinética de la glucosa, junto con cambios en múltiples mecanismos reguladores de la glucemia, como los niveles de hormonas gastrointestinales y pancreáticas relacionados con la homeostasis de la glucosa'. Esta revisión pretende describir las características de la HHP y actualizar la información disponible hasta la fecha sobre esta entidad.

\section{Definición}

La hipoglucemia hiperinsulinémica postprandial (HHP), también denominada por algunos autores síndrome de dumping tardío ${ }^{8}$, es una condición caracterizada por síntomas de hipoglucemia que ocurren 1-3 horas (h) después de la ingesta, acompañada de bajos valores de glucosa plasmática, precedidos típicamente por un elevado nivel de glucosa y una alta concentración de insulina.

\section{Síntomas de hipoglucemia}

El diagnóstico clínico es poco sensible y específico. Puede resultar difícil distinguir los síntomas de HHP de los del síndrome de dumping?. Para diferenciar ambas entidades, algunos autores los denominaron dumping precoz y tardío. El dumping precoz ocurre frecuentemente luego del BGYR, aparece 10-30 minutos ( $\mathrm{min}$ ) luego de una ingesta de alta densidad calórica, en particular de hidratos o grasas, atribuido a la llegada rápida del contenido hiperosmolar al intestino distal con paso de fluidos al intestino y liberación de hormonas intestinales. Presenta síntomas digestivos (náuseas, vómitos, meteorismo, dolor de tipo cólico y diarrea) y vasomotores (sudoración, palpitaciones), pero no síntomas de neuroglucopenia dado que no se asocia a hipoglucemia. En cambio, la HHP o dumping tardío ocurre luego de uno o más años (hasta 10) de la $C B$, se presenta entre 1 y $3 \mathrm{~h}$ después de la ingesta y se acompaña de síntomas de hipoglucemia. Éstos se categorizan como síntomas autonómicos que incluyen ansiedad, sudoración, temblores y palpitaciones, y síntomas neuroglucopénicos como confusión, debilidad, cefalea, mareos, visión borrosa, desorientación, alteraciones del habla y deterioro del sensorio ${ }^{4,8}$. La hipoglucemia persistente no diagnosticada puede progresar a pérdida de la conciencia, convulsiones, coma e incluso la muerte. En los pacientes operados de BGYR se ha descripto una mayor tasa de muertes por accidentes; algunos autores especulan que podría ser el resultado de hipoglucemias severas desapercibidas?

\section{Diagnóstico}

Como criterio clínico se utiliza la tríada de Whipple. La Asociación Americana de Diabetes (ADA) define actualmente a la hipoglucemia grave o clínicamente significativa cuando la glucemia es inferior a $54 \mathrm{mg} / \mathrm{dl}(3,9 \mathrm{mmol} / \mathrm{l})$. En los estudios realizados sobre HHP se utilizaron puntos de corte entre 45 a $60 \mathrm{mg} / \mathrm{dl}$ para definir la hipoglucemia. Para diagnosticar la HHP se necesita una historia clínica y nutricional detallada, y un alto nivel de 
sospecha. El interrogatorio minucioso es fundamental para arribar al diagnóstico; debe preguntarse sobre hábitos alimentarios, tipo y horarios de comidas, síntomas específicos de hipoglucemia y su relación temporal con la ingesta. En los pacientes que presentan hipoglucemia en ayunas deben descartarse otras causas".

Un estudio demostró que los síntomas no correlacionaron con los valores de glucemia, y que los pacientes asintomáticos presentaron valores aún más bajos de glucemia que los sintomáticos luego de un test de comida mixta (43 vs $54 \mathrm{mg} / \mathrm{dll}$ ), al igual que tampoco hubo correlación entre los valores de insulina y glucagón, y la aparición de síntomas ${ }^{9}$. Aún se desconoce por qué algunos pacientes desarrollan síntomas de neuroglucopenia mientras que otros con iguales valores de glucemia permanecen asintomáticos. Habría diferentes umbrales de percepción de la hipoglucemia o mecanismos adaptativos, tal vez modificados por factores estresores. Se postulan diferencias en la captación de la glucosa y utilización en áreas del cerebro que promueven neuroglucopenia. El factor que más correlaciona con la intensidad de los síntomas sería el grado de variación de la glucemia, más importante que el nadir de glucemia alcanzado ${ }^{10}$.

El diagnóstico de HHP se realiza con niveles de glucemia menores a $55 \mathrm{mg} / \mathrm{dl}$ junto con insulinemia de al menos $3 \mathrm{uU} / \mathrm{ml}$ y péptido-C de al menos $0,6 \mathrm{ng} / \mathrm{ml}$, en ausencia de secretagogos en sangre. Los niveles de proinsulina están elevados en la hiperinsulinemia endógena (no así en la administración exógena de insulina donde también los niveles de péptido $\mathrm{C}$ son indetectables o muy bajos). Estos niveles son significativamente mayores en el caso de insulinoma comparado con nesidioblastosis, aunque pocos estudios refieren los valores de proinsulina ${ }^{10}$.

El diagnóstico de hipoglucemia puede realizarse con automonitoreo o a través de pruebas provocativas, como la prueba de tolerancia oral a la glucosa (PTOG) o la prueba de comida mixta. La PTOG puede provocar hipoglucemia en 10-12,5\% de los sujetos sanos ${ }^{11}$, y dado que la ingesta es de consistencia líquida llegaría más rápido al intestino distal y podría dar síntomas de dumping precoz, por lo tanto se prefieren otras pruebas diagnósticas. La prueba de tolerancia a una comida mixta es una prueba de provocación en la cual se administra una comida estandarizada en proporción de proteínas, grasas y carbohidratos.
Los niveles de glucosa e insulina se miden en ayunas y cada 30 min después de la comida durante $3 \mathrm{~h}$. Se considera un test positivo cuando se observan valores de glucemia normal en ayunas, hiperglucemia precoz, hiperinsulinemia previa a la hipoglucemia y valores de glucosa plasmática entre 50 y $60 \mathrm{mg} / \mathrm{dl}$ asociados a síntomas de hipoglucemia9. El monitoreo continuo de glucosa (MCG) puede ser una herramienta útil en el diagnóstico de la variabilidad glucémica en condiciones de vida real (con variaciones en la porción, la composición de nutrientes, la actividad física y la respuesta de hormonas contrarregulatorias) frente a un test de tolerancia a comida mixta en el contexto de investigación. En un estudio con un número reducido de pacientes, el MCG mostró una sensibilidad diagnóstica de $90 \%$ y una especificidad de $50 \%$, versus 33 y $44 \%$ respectivamente para el test de comida mixta ${ }^{12}$. El MCG evidenció que los pacientes que presentaron síntomas de neuroglucopenia post BGYR estuvieron durante un promedio de $63 \mathrm{~min}$ diarios con niveles menores a $70 \mathrm{mg} / \mathrm{dl}$ de glucosa intersticial, y 30 min con valores menores a $60 \mathrm{mg} / \mathrm{dl}$. Además se detectaron valores de hipoglucemia en el $50-70 \%$ de los pacientes asintomáticos ${ }^{13-14}$. Otros autores mostraron con el MCG durante cinco días (versus un grupo control de pacientes obesos en los que no se detectaron episodios de hipoglucemia) que el $75 \%$ de los pacientes operados de BGYR (a 5-8 años de la cirugía) experimentó episodios de hipoglucemia $<55$ $\mathrm{mg} / \mathrm{dl}$ ) y el $38 \%$ sufrió episodios de hipoglucemia nocturnos hasta $3 \mathrm{~h}$ luego de la ingesta, mientras que con el test de comida mixta se hizo el diagnóstico de hipoglucemia postprandial en el 29\% de los pacientes ${ }^{15}$. Hay que tener en cuenta que con valores en el rango bajo de hipoglucemia, la glucosa intersticial puede ser 20 mg/dl más alta que la plasmática ${ }^{16}$.

\section{Diagnóstico diferencial y factores de riesgo para el desarrollo de HHP}

Varias causas de hipoglucemia postprandial se han descripto en los pacientes luego de la cirugía bariátrica, incluyendo dumping tardío, síndrome de nesidioblastosis, hipoglucemia facticia e insulinoma. El diagnóstico correcto es esencial, sobre todo por la complejidad y posible co-ocurrencia de estas condiciones ${ }^{17}$. En la Tabla 1 se describen los diagnósticos diferenciales.

Se consideran factores de riesgo para el desa- 
rrollo de HHP: sexo femenino, síntomas de hipoglucemia previos a la cirugía, años transcurridos desde la cirugía y no haber padecido diabetes antes de la cirugía ${ }^{18}$. Otros autores observaron como factores de riesgo: hemoglobina glicosilada (HbA1c) e índice de masa corporal (IMC) más bajos previos a la cirugía y mayor pérdida de peso posterior a la misma ${ }^{19-20}$. Los pacientes con diabetes tipo 2 (DM2) previo a la cirugía tendrían un factor protector para el desarrollo de HHP, ya sea por disminución de la reserva pancreática, mayor insulinorresistencia periférica, o ambas ${ }^{18,20}$.

Para detectar la hipoglucemia facticia debe dosarse péptido- $C$ en sangre (debe ser menor a $0,6 \mathrm{ng} / \mathrm{ml}$ ) y buscar la presencia de sulfonilureas en sangre $u$ orina $^{18}$. Un nivel elevado de péptido-C postprandial excluye el diagnóstico de hiperinsulinemia facticia por insulina, mientras que los secretagogos (sulfonilureas y metiglinidas) producen aumento tanto de insulina como de péptido- $\mathrm{C}^{20}$.

Un test de ayuno de $72 \mathrm{~h}$ puede ser necesario para descartar la presencia de insulinoma. Un estudio en pacientes con insulinoma describió hipoglucemia únicamente de ayuno en el $56 \%$ de los pacientes, hipoglucemia sólo postprandial en el $22 \%$ y la combinación de ambas en el otro $22 \%{ }^{21}$. La detección de insulinomas y tumores pancreáticos neuroendocrinos post BGYR se publicó en una decena de $\operatorname{casos}^{22}$. El dosaje de proinsulina ayuda en el diagnóstico diferencial de hiperinsulinemia endógena por insulinoma (proinsulina mayor a $5 \mathrm{pmol} / \mathrm{l}$ ) o por nesidioblastosis (menor a $5 \mathrm{pmol} / \mathrm{l})$; en el caso de insulinoma también se observa la supresión de $\beta$-hidroxibutirato ${ }^{21}$. Las imágenes -ultrasonografía endoscópica, tomografía computada, resonancia nuclear magnética o tomografía por emisión de positrones- pueden ser útiles para identificar un insulinoma ${ }^{23}$. Con la ecoendoscopía, luego del BGYR, puede resultar complejo acceder al duodeno para visualizar la cabeza del páncreas, aunque se ha descripto que la punción biopsia a través del pouch gástrico es segura ${ }^{21}$. La arteriografía selectiva con estimulación intraarterial de calcio es útil para localizar el área de células $\beta$ hiperfuncionantes, más difuso en la nesidioblastosis, y localizado en la presencia de insulinoma ${ }^{24-25-26}$. Puede realizarse tatuaje de la lesión por vía ecoendoscópica que permite cirugías mínimamente invasivas con disminución de los riesgos. El tratamiento es la pancreatectomía parcial o total según la localización (cercanía con el conducto biliar común) o en lesiones múltiples²1.

La confirmación diagnóstica requiere de la anatomía patológica. La nesidioblastosis presenta hiperplasia de las células $\beta$, desarrollo de células $\beta$ a partir de los ductos pancreáticos y aumento del número de los islotes. Existe controversia sobre el diagnóstico de nesidioblastosis en los adultos; algunos lo consideran una variante de la anatomía normal dado que se han descripto hallazgos similares en autopsias de pacientes sin antecedentes de hipoglucemia. Por otro lado la pancreatectomía parcial falló en resolver la HHP en varios casos, por lo cual se sugiere que otros mecanismos podrían estar involucrados en la hipoglucemia postprandial ${ }^{27}$.

\begin{tabular}{|l|}
\hline Causas endógenas \\
\hline Insulinoma \\
\hline Síndrome de dumping precoz \\
\hline Hipoglucemia hiperinsulinémica postprandial \\
\hline Causas exógenas \\
\hline $\begin{array}{l}\text { Uso de hipoglucemiantes orales secretagogos } \\
\text { (sulfonilureas, metiglinidas) }\end{array}$ \\
\hline Administración exógena de insulina \\
\hline
\end{tabular}

Tabla 1: Diagnóstico diferencial de hipoglucemia post BGYR'.

\section{Prevalencia}

Existe gran variación en los datos de prevalencia de HHP y se desconoce con exactitud porque la entidad es subdiagnosticada. Algunos estudios refieren hipoglucemia autoreferida por pacientes luego del BGYR a través de cuestionarios en 0,2$0,36 \%$, aunque otros sugieren que la prevalencia sería mucho mayor y que hasta un tercio de los pacientes podría sufrirla en algún momento de la evolución postoperatoria. Un estudio sueco con 5.040 pacientes observó hipoglucemias con síntomas graves que requirieron hospitalización en el $0,2 \%$ de los casos, con un tiempo de seguimiento promedio de 32,4 meses $^{28}$. Otros estudios expresaron una prevalencia de $10-75 \%$ variable según la herramienta diagnóstica utilizada, el diseño del estudio, el punto de corte usado para definir hipoglucemia y el tiempo de seguimiento posterior a la $\mathrm{CB}^{29}$. En un estudio con 1.206 pacientes se detectó HHP en 2,7\% al año de la cirugía de BGYR y en $13,3 \%$ a los cinco años de seguimiento ${ }^{20}$. 


\section{Fisiopatología}

A partir de la descripción de una serie de casos de nesidioblastosis post BGYR en el año 2005, de Service et al. ${ }^{27}$, se interpretó que ésta podría ser la causa de la HPP y se denominó síndrome de hipoglucemia pancreatogénica no insulinoma. La nesidioblastosis del adulto con hiperplasia de las células $\beta$ es rara, y se describió por primera vez en 1975 ${ }^{30-31}$. Ya se habían descripto episodios de hipoglucemia hiperinsulinémica en aproximadamente el $5 \%$ de pacientes gastrectomizados por diversas causas (úlcera péptica o cáncer) ${ }^{32}$. En los casos iniciales de HHP descriptos se sugirió que los cambios histopatológicos pancreáticos eran característicos de nesidioblastosis y que podían atribuirse al estímulo prolongado de la hipersecreción de GLP-1: hipertrofia de las células $\beta$, hiperplasia de los islotes y neoformación de islotes de Langerhans a partir del epitelio ductal ${ }^{33}$. Pero, años más tarde, la revisión de los casos de Service et al. no confirmó el diagnóstico de nesidioblastosis; se interpretó entonces que la hipertrofia de las células $\beta$ que acompaña el aumento del IMC persistía luego del BGYR, con hiperfunción de las células $\beta$ a pesar del descenso de peso con la cirugía ${ }^{34,35}$. Sin embargo otros autores describieron que la HHP no se acompaña de hiperplasia de los islotes o de hiperactividad de la célula $\beta^{35}$. Meier detalló aumento del diámetro nuclear de las células $\beta$ sugestivo de incremento de la actividad secretora que correspondería al IMC aumentado antes del BGYR ${ }^{35}$. Si bien no se conoce con precisión el o los mecanismos causales de la HHP, la exclusión del intestino proximal con la consiguiente llegada rápida de alimentos al intestino distal sería un factor importante. Se describió una absorción acelerada de glucosa marcada, con un pico más alto y nadir más bajo de glucemia en los pacientes operados de BGYR que presentaron síntomas versus los asintomáticos. Luego del BGYR se observó un pico de glucosa alto y precoz en sangre (frecuentemente mayor a $200 \mathrm{mg} / \mathrm{dl}$ ), seguido de una respuesta exagerada de GLP-1 e insulina. Goldfine et al. refirieron un aumento de 5-10 veces de GLP-1 postprandial en los pacientes operados de BGYR ${ }^{36}$.

La HHP no está causada necesariamente por la función autónoma de las células $\beta$, sino que es una respuesta exagerada a una carga oral de glucosa. Se propusieron varios mecanismos como causa posible de la HHP: aumento de las increti- nas GLP-1 y GIP, incremento de la sensibilidad de la célula- $\beta$ al GLP-1, persistencia del incremento de la masa $\beta$ celular o de su función post BGYR que sería adecuada para el paciente obeso previamente a la cirugía, pero excesiva para el paciente que disminuyó su masa grasa. En contra de este último argumento estaría el hecho de que la HHP no se presenta hasta el año posterior a la cirugía, aunque el paciente reduce drásticamente la ingesta luego de la misma, y que la HHP no se observa luego de procedimientos puramente restrictivos como la banda gástrica ajustable 27. Por otro lado, la reducción de la masa $\beta$ celular con la pancreatectomía parcial tampoco resuelve la HHP en todos los casos $^{36}$. Otros mecanismos posibles serían la mejoría de la insulinosensibilidad atribuible a la reducción de peso, secreción inapropiada de insulina, factores genéticos, alteración en otras hormonas gastrointestinales (grelina, péptido YY, leptina) u otras adaptaciones de la mucosa intestinal que puedan influir en la absorción de glucosa y otros nutrientes y afectar las respuestas en magnitud del eje intestino-hígado-cerebro ${ }^{34}$, aumento de adiponectina ${ }^{37}$, respuesta anormal o atenuada de las hormonas contrarregulatorias ${ }^{38}$, disminución de depósitos de glucógeno o de sustratos para la gluconeogénesis ${ }^{39}$. Además de diferencias en la sensibilidad de la célula $\beta$ al GLP-1, podrían haber diferencias en los efectos extrapancreáticos del GLP-1 sobre el cerebro, el hígado, el tracto gastrointestinal y/o el nervio vago ${ }^{18}$.

El aumento de GLP-1 postprandial luego del BGYR, que es considerado uno de los factores importantes en la remisión de la DM2 post cirugía, podría también ser la causa de la hipoglucemia reactiva o HHP. Los niveles de GLP-1 postprandiales son más altos en los pacientes que presentan síntomas comparados con pacientes asintomáticos. El glucagón postprandial se encuentra alto en pacientes operados de BGYR y con un antagonista del receptor de GLP-1, Exendina 9-39, aumenta aún más ${ }^{40}$. La disfunción de la célula $\alpha$ del páncreas podría tener un rol en la HHP dado que el aumento del glucagón podría atribuirse a un origen intestinal: las células $L$ del intestino distal secretan pre-proglucagón a partir del cual se genera GLP-1 y glucagón, junto con otras moléculas: GLP-1, GLP-2, glicentina y oxintomodulina ${ }^{8,18}$.

El Gráfico 1, adaptado de Patti et al., resume los mecanismos propuestos como causales de la $\mathrm{HHP}^{48}$. 


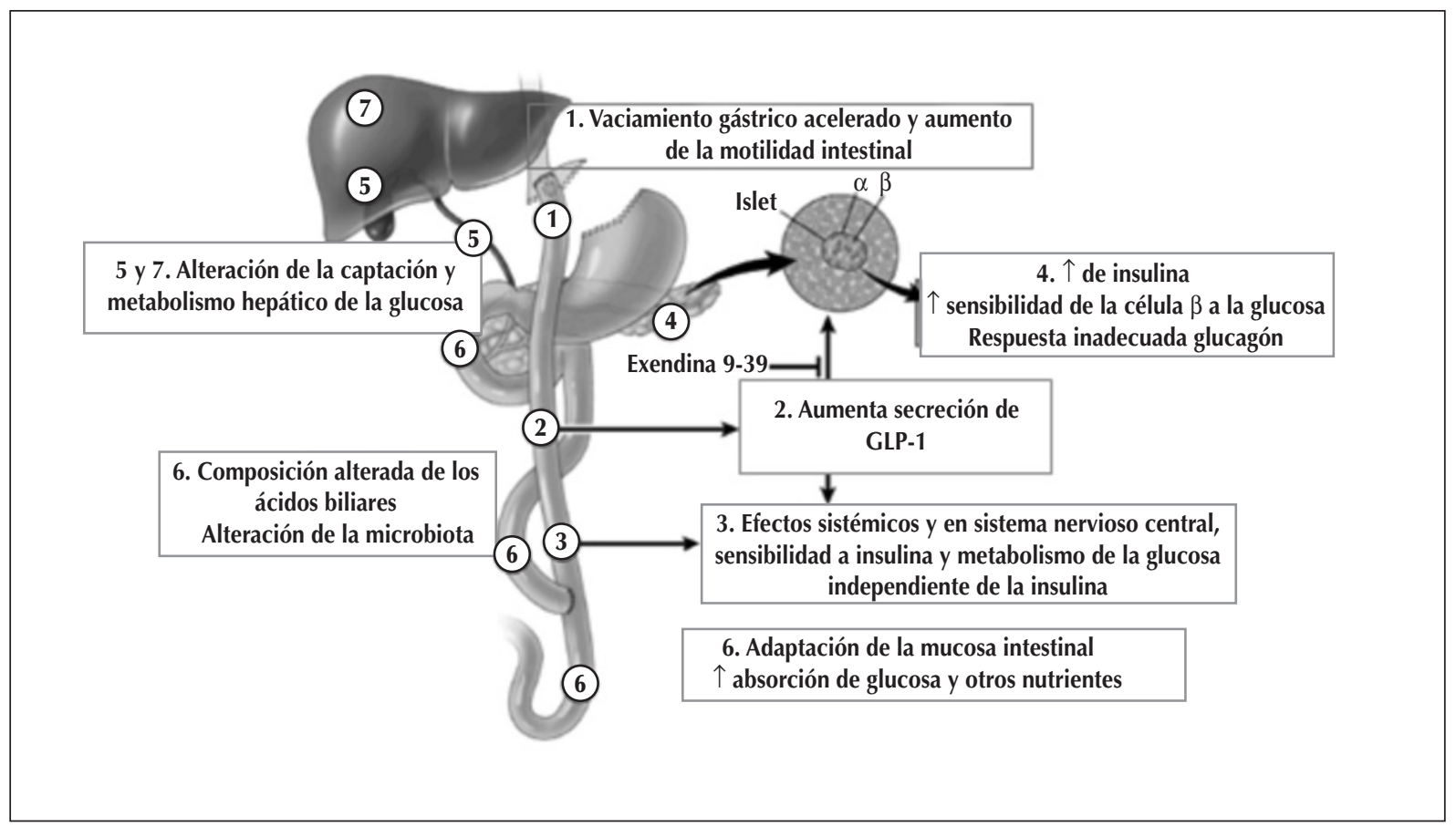

Gráfico 1: Mecanismos causales de HHP.

\section{Tratamiento de la HHP}

Dado que no existe consenso sobre la historia natural y la fisiopatología de la HHP, no hay guías terapéuticas aceptadas para su tratamiento ${ }^{41}$. El objetivo terapéutico es moderar las excursiones postprandiales de la glucosa plasmática, reducir la secreción de insulina y controlar de este modo los episodios de hipoglucemia.

\section{Tratamiento médico}

\section{Dieta}

La mayoría de los casos de HHP se relaciona con transgresiones alimentarias. Se debe educar a los pacientes a consumir porciones pequeñas y frecuentes con hidratos de carbono de bajo índice glucémico. La comida mixta con proteínas, huevos, carne de pollo, cerdo, vaca, pescados y mariscos, jamón, pastrón o pavita, aceite de oliva, palta, aceitunas y alimentos con alto contenido de fibra manifestó niveles de insulina más bajos, sin riesgo de hipoglucemia postprandial. Debe tenerse en cuenta que la amplia mayoría de los alimentos procesados tiene jarabe de maíz de alta fructosa que produce una respuesta dual debido a la diferente absorción de los monosacáridos que lo componen (glucosa y fructosa). La glucosa se absorbe velozmente y genera una rápida liberación de insulina, mientras que la fructosa se absorbe lentamente a través del transportador GLUT-5, lo cual puede generar una hipoglucemia posterior dada la disociación entre los altos niveles de insulina (post carga de glucosa) y la lenta absorción de la fructosa ${ }^{42}$.

En diversos trabajos, con un número reducido de pacientes, se observó que las modificaciones en la dieta logran la mejoría de los síntomas en el $83 \%$ de los pacientes y la resolución completa en el $25 \%$ de los casos. Kellogg et al. demostraron que puede evitarse la respuesta hiperinsulinémica postprandial con una dieta baja en hidratos de carbono simples, y rica en proteínas y fibra ${ }^{8}$. Algunos autores sugieren limitar la cantidad de carbohidratos a 15-30 g por comida, sin el agregado de azúcares simples ${ }^{43}$. Un problema frecuente es la baja adherencia de los pacientes en el largo plazo a una dieta restringida en hidratos de carbono. En esos casos se indica buscar alternativas terapéuticas.

\section{Fármacos}

El uso de medicación se utilizó en pacientes con HHP refractaria a las modificaciones de la dieta, en series de número reducido y en reporte de casos.

- Acarbosa: es un inhibidor de la $\alpha$-glucosidasa. Disminuye la absorción intestinal de carbohidratos al interferir con las enzimas del ribete en cepillo que degradan polisacáridos a monosacáridos, atenúa así el aumento postprandial de la glucosa e insulina. En pacientes con BGYR la toma de acor- 
bosa 15 min luego de la ingesta reduce los niveles de GLP-1 e insulina, y disminuye los síntomas de hipoglucemia ${ }^{44}$. Los efectos adversos comunes de la acarbosa (diarrea, flatulencias, dolor y distensión abdominal) pueden limitar su uso. Para minimizarlos se sugiere comenzar con dosis bajas de $25 \mathrm{mg}$ en una comida diaria durante una semana y progresar la dosis hasta 50-100 mg con cada comida ${ }^{45}$.

- Diazóxido: es un agonista de los canales de potasio ATP-dependientes de las células $\beta$ y reduce la secreción de insulina. Algunos autores la consideran la droga de primera elección en casos de HHP. Los efectos adversos posibles son hipotensión, retención de líquidos, hirsutismo, mareos, cefalea, ansiedad, insomnio, anorexia y diarrea ${ }^{20,43}$.

- Octreotide o pasireotide: son análogos de la somatostatina. Pueden mejorar los síntomas de la HHP por la suma de varios efectos: retrasan el tránsito intestinal, inhiben la secreción de hormonas gastrointestinales, incretinas e insulina y la vasodilatación postprandial del lecho esplácnico ${ }^{43,46}$.

El uso de insulinas de acción rápida previas a la ingesta no fue efectivo para reducir el pico de hiperglucemia e insulinemia y produjo nadir más bajo de glucemia postprandial.

El uso experimental de Exendina 9-39, un inhibidor del GLP-1, logró reducir la hiperinsulinemia postprandial y previno la $\mathrm{HHP}^{40}$. En un trabajo reciente, el uso de Exendina 9-39 por vía subcutánea en nueve pacientes con HHP refractarias a las modificaciones de la dieta elevó el nadir de la glucemia en $66 \%$, disminuyó el pico de insulina y se redujeron los síntomas de neuroglucopenia en un $80 \%$, sin efectos adversos ${ }^{47}$. Existen diferencias sustanciales en la respuesta del tratamiento con Exendina 9-39, lo que sugiere variabilidad interindividual en la contribución relativa de la secreción de incretinas, función secretora de los islotes y otros factores metabólicos en pacientes con $\mathrm{HHP}^{48}$.

Es interesante que el tratamiento con agonistas de GLP-1, liraglutida o exenatide mejoró los síntomas de hipoglucemia en cinco pacientes y disminuyó las excursiones de glucosa intersticial medidas por MCG. La activación más persistente del GLP-1R por la administración exógena de GLP-1 activaría la respuesta del glucagón con estabilización de la glucosa intersticial ${ }^{49}$.

También, en algunos casos, se usaron bloqueantes cálcicos: verapamilo o nifedipina para reducir la secreción de insulina glucosa dependiente. Los efectos adversos más frecuentes son: cefalea, trastornos gastrointestinales, edemas periféricos e hipotensión arterial ${ }^{20,50}$.

\section{Tratamiento quirúrgico}

La pancreatectomía o reversión del BGYR es el último recurso que debe considerarse en pacientes con síntomas debilitantes de neuroglucopenia y que han sido refractarios al tratamiento dietético y farmacológico.

Un algoritmo diagnóstico antes de optar por el tratamiento quirúrgico debería incluir un test negativo de ayuno de $72 \mathrm{~h}$, monitoreo continuo de glucosa e imágenes para descartar la secreción de insulina autónoma por un insulinoma. En estos casos, el péptido-C y la insulinemia se encuentran inadecuadamente altos para los valores de glucemia ${ }^{51,52}$.

La elección del tratamiento quirúrgico debe considerar los riesgos perioperatorios, la posibilidad de reganancia de peso y sus consecuencias sobre las comorbilidades asociadas a la obesidad. En una revisión que incluyó 75 pacientes con HPP, en $68 \%$ se realizó pancreatectomía, en $23 \%$ reversión del BGYR y en 15\% resección del pouch gástrico. En $11 \%$ se efectuaron dos o más procedimientos consecutivos y en varios pacientes intervenciones combinadas. La resolución de los síntomas se logró en el 67-82\% de los casos, por lo cual es fundamental dilucidar los mecanismos de la HHP para definir un tratamiento adecuado que controle la variabilidad glucémica postprandial ${ }^{53}$. Al asumir que el vaciamiento gástrico acelerado contribuye a la HHP, se propuso aumentar la restricción o reducir la velocidad del tránsito como una alternativa terapéutica ${ }^{54}$, ya sea a través de una aplicación endoscópica del pouch gástrico, de restricción con una banda gástrica ajustable o un anillo de silastic sobre el pouch gástrico ${ }^{5,52}$. No se conocen los resultados en el largo plazo o la morbilidad vinculada a la banda en estos casos.

La reversión del BGYR se describió por primera vez en 2006 con el objetivo de restaurar la función del píloro y la continuidad duodenal ${ }^{53,55}$. En una revisión de 100 pacientes, la HHP fue la segunda causa $(8,5 \%)$ de reversión del BGYR luego de la desnutrición severa $(12,3 \%)$ y el síndrome de dumping severo $(9,4 \%)$, aunque varios estudios no distinguen entre dumping precoz y tardío, por lo que la HHP podría llegar a ser la primera causa de reversión de la cirugía ${ }^{56}$. En algunos pacientes se agregó la gastrectomía en manga para prevenir la reganancia de peso, aunque ésta agrega 
morbilidad quirúrgica y existen reportes de casos con número reducido de pacientes que proponen otras técnicas para la reversión del BGYR ${ }^{57}$. Con la alimentación a través de una gastrostomía del estómago remanente (el excluido del tránsito de los alimentos) pueden evitarse los síntomas de hipoglucemia en algunos pacientes; este sería un método útil para identificar aquellos que puedan beneficiarse de la reversión del BGYR ${ }^{52,58,59}$.

Existen reportes de pancreatectomía parcial y aún total para controlar la HHP. No está definida la extensión adecuada de la resección y existe el riesgo de desarrollar DM insulinodependiente e insufi- ciencia pancreática exocrina. La estimulación arterial selectiva con calcio ayudaría a identificar áreas hipersecretoras del páncreas y reducir la extensión de la resección ${ }^{60-61}$. Si bien con la pancreatectomía parcial el $75 \%$ de los pacientes mejoró los síntomas y la calidad de vida, en el resto hubo persistencia de los síntomas; algunos sujetos mejoraron inicialmente pero entre el 60 al $90 \%$ de los pacientes tuvieron recurrencia de la hipoglucemia seguidos en el largo plazo (cuatro años en promedio) ${ }^{60}$.

Luego de la revisión de la bibliografía, y según nuestra experiencia, proponemos el siguiente algoritmo terapéutico (Gráfico 2).

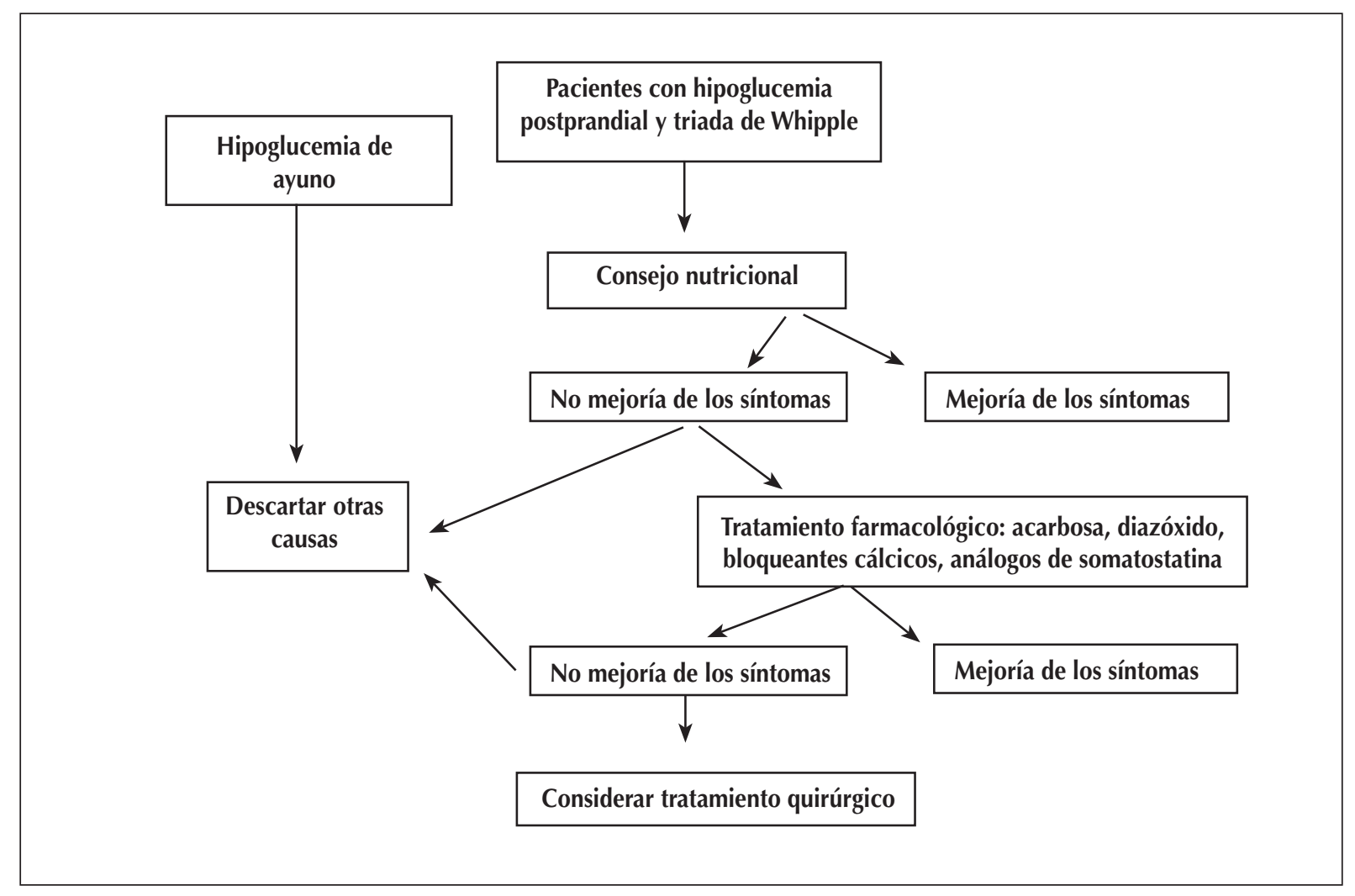

Gráfico 2: Algoritmo terapéutico sugerido de la HHP.

Debemos conocer mejor la fisiopatología de la HHP y los resultados de los tratamientos en el largo plazo para individualizar el tratamiento adecuado e informar a los pacientes sobre los posibles riesgos y consecuencias de los mismos.

\section{Hipoglucemia luego de la cirugía bariátrica en pacientes con DM1}

En los últimos años se publicaron artículos con series de casos donde se realizó CB a pacientes con DM1 y obesidad severa ${ }^{62}$. Algunos tuvieron episodios de hipoglucemia en los primeros meses del postoperatorio lo cuales se atribuyeron a la inadecuación del esquema de insulina exógena ${ }^{63,64}$. Se propone que los pacientes utilicen insulinas rápidas postprandiales en dosis adecuadas a la ingesta de hidratos de carbono tolerada a fin de evitar hipoglucemias postprandiales ${ }^{65}$. 


\section{Casos clínicos}

Presentamos tres casos clínicos de pacientes que no tuvieron DM2 antes de la cirugía y desarrollaron HHP post CB de distinta etiología.

\section{Caso clínico 1}

Paciente de sexo femenino, de 54 años de edad, con antecedentes de BGYR por obesidad mórbida (IMC $41 \mathrm{~kg} / \mathrm{m}^{2}$ ) dos años previos al episodio actual (con peso: $62 \mathrm{~kg}$, IMC 25,5 kg/m²). Sufrío convulsión tónico-clónica generalizada durante el sueño, quedando posteriormente en coma. Fue llevada de urgencia por el esposo a una guardia donde se constató glucemia de $43 \mathrm{mg} / \mathrm{dl}$, con hemograma (hematocrito 40,5\%, hemoglobina $13,6 \mathrm{~g} / \mathrm{dl}$, glóbulos blancos $6.540 \mathrm{~mm}^{3}$ con $58 \%$ de neutrófilos y $32 \%$ de linfocitos), hepatograma (amino asparto transferasa -AST-) $43 \mathrm{UI} / \mathrm{l}$, alanina aminotransferasa (ALA) 37UI/l, función renal (urea $16 \mathrm{mg} / \mathrm{dL}$, creatinina $0,8 \mathrm{mg} / \mathrm{dL}$ ) y gases en sangre normales $(\mathrm{pH} 7,40$, bicarbonato $26,6 \mathrm{mEq} / \mathrm{L}$, exceso de base 1,5). Recibió suero glucosado hipertónico al 50\% recuperando el estado del sensorio, con amnesia de lo ocurrido. Fue dada de alta al día siguiente encontrándose lúcida, orientada, sin signos de foco neurológico, con glucemias de control alrededor de $90 \mathrm{mg} / \mathrm{dl}$ y con indicación de consulta urgente con un diabetólogo.

El episodio de convulsiones y coma generó gran ansiedad y temor en la paciente y su familia. La paciente no había consultado por síntomas de hipoglucemia previamente a este episodio, aunque interrogándola retrospectivamente en forma detallada, refirió malestar inespecífico con somnolencia y sudoración postprandial en alguna oportunidad. La cena previa al episodio de convulsiones y coma había sido de pastas sin el agregado de carnes o verduras. No había ingerido postre ni alcohol durante la comida.

Se le dieron instrucciones dietéticas precisas para evitar el consumo de azúcares simples y alimentos de alto índice glucémico, y se le proveyó un glucómetro para que realizara controles ante cualquier síntoma sospechoso de hipoglucemia. La paciente tuvo muy buena adherencia a las indicaciones de la dieta. A pesar de varios controles en distintas momentos en situación postprandial, no repitió episodios de hipoglucemia durante los siguientes 12 meses de seguimiento cercano.

Este caso muestra que la intensidad de los síntomas no correlaciona con la intensidad del tratamiento y que la adherencia a las indicaciones dietéticas puede prevenir el desarrollo de HHP.

\section{Caso clínico 2}

Paciente de sexo masculino, de 43 años de edad. Historia de 15 años de obesidad con IMC 40,6, con dificultad en el descenso de peso por sensación de "hambre permanente" sin registro de saciedad. Laboratorio previo a la CB: glucemia $91 \mathrm{mg} / \mathrm{dl}$, colesterol total $222 \mathrm{mg} / \mathrm{dl}$, vitamina D $23 \mathrm{ng} / \mathrm{ml}, \mathrm{TSH}$ 0,91 uU/ml, insulinemia 21,2 uU/ml, HA1C 5.1\% HOMA 4.7.

Tres días después de realizarse una GVM consultó a la guardia por episodios de mareos con trastornos del habla. Se detectó glucemia de $40 \mathrm{mg} / \mathrm{dl}$, recibió suero glucosado y fue externado con controles de glucemia y pautas alimentarias. El paciente refierió síntomas de neuroglucopenia, sin diarrea, dolor abdominal, ni otros síntomas vasomotores. Persistió con hipoglucemias diurnas y nocturnas que requirieron ingestas cada 2-3 h para prevenirlas. A los 15 días de la cirugía se detectó palidez, sudoración y glucemia de $32 \mathrm{mg} / \mathrm{dl}$ (con ayuno de 2,5 h) que revirtió rápidamente luego de la ingesta. Se indicó laboratorio: glucemia con $4 \mathrm{~h}$ de ayuno $65 \mathrm{mg} / \mathrm{dl}$, insulinemia 10-15 uU/ml (inapropiadamente elevada para la glucemia), péptido C $1 \mathrm{ng} / \mathrm{ml}$. Se asumió como hipoglucemia hiperinsulinémica. Se realizó PTOG que fue negativa para hipoglucemia reactiva.

Por episodios de hipoglucemia de ayuno, se interpretó como probable insulinoma. Se realizó RNM con gadolinio: informó imagen nodular de $3 \mathrm{~cm}$ con contornos bien definidos en la cola del páncreas, con realce homogéneo con el contraste. A los tres meses de la CB se indicó pancreatectomía distal por vía laparoscópica y el paciente quedó libre de síntomas. La anatomía patológica informó neoplasia endocrina.

Al reinterrogar al paciente refirió que previamente a la cirugía tenía parestesias peribucales y la urgencia por comer cuando realizaba dietas hipocalóricas. Ese síntoma en cierto modo limitaba su adherencia a los planes alimentarios indicados.

Este caso muestra que las hipoglucemias de ayuno, inmediatas a la cirugía, su persistencia a pesar del manejo nutricional y la ausencia de síntomas vasomotores y gastrointestinales, plantean la necesidad de evaluar otras causas.

\section{Caso clínico 3}

Paciente de sexo femenino, de 38 años de edad, con $51 \mathrm{~kg}$ e IMC 19,5 kg/m² (previo a la ciru- 
gía: IMC $\left.47,5 \mathrm{~kg} / \mathrm{m}^{2}\right)$, operada de BGYR dos años y medio antes de los síntomas de hipoglucemia. Tuvo varios episodios de hipoglucemia siempre postprandiales, con valores que oscilaron entre 35-42 mg/dl. Durante la realización de una PTOG la paciente sufrió una convulsión generalizada. La paciente no cumplía adecuadamente con el plan alimentario indicado y repitió episodios de hipoglucemia luego de la ingesta de azúcares simples. Surgieron de la historia y del nuevo interrogatorio antecedentes de bulimia e intentos de suicidio previos a la cirugía, datos que no habían sido detectados con anterioridad.

El dosaje de insulinemia basal fue de 1,8uU, a los $120 \mathrm{~min} 73 \mathrm{uU}$ y a los $180 \mathrm{~min} 4,34 u U$. Se interpretó el cuadro como hipoglucemia hiperinsulinémica postprandial post BGYR. Inicialmente se medicó con diazóxido 100 a 200 mg que debió ser suspendido por toxicidad renal; posteriormente se la medicó con octreotide 30 mg por vía intramuscular (IM) (Sandostatin LAR 30) sin buena respuesta. Con verapamilo en dosis progresivas y de liberación prolongada mejoró sólo parcialmente. Se le realizó una gastrostomía del estómago residual (excluido del tránsito de los alimentos) con lo cual se controlaron los episodios de hipoglucemia. Se planteó en ese momento la reversión del BGYR a lo cual la paciente se negó.

Durante los meses de seguimiento tuvo un episodio de pérdida de la conciencia y traumatismo craneoencefálico atribuido a neuroglucopenia. Se le realizó RNM y arteriografía selectiva con estimulación intraarterial de calcio para descartar la presencia de insulinoma; los resultados sugirieron la posibilidad de nesidioblastosis pancreática.

Finalmente, y ante la persistencia de los síntomas, se decidió realizar tratamiento quirúrgico de la HHP; a los cuatro años del BGYR se realizó esplenopancreatectomía corpocaudal. La anatomía patológica informó: nesidioblastosis con aumento del número de islotes de distintos tamaños y forma, algunos islotes septales (inmersos en tabiques fibrosos glandulares) con proliferación ductal (complejos ductulo insulares), en algunos ductos de mayor calibre se reconoció proliferación focal y leve atipía del epitelio columnar.

La paciente persistió con episodios de hipoglucemia graves hasta el coma. A los siete meses de la pancreatectomía parcial y ante la recidiva de los síntomas, se realizó duodenopancreatectomía cefálica. Tuvo una evolución postoperatoria tórpida, requirió expansión con cristaloides por tendencia a la hipotensión y presentó episodios de hipoglucemia que fueron corregidos con suero glucosado hipertónico. Manifestó como complicación dehiscencia de la anastomosis bilioentérica, por lo que debió ser re intervenida a la semana de la cirugía. Estuvo con nutrición parenteral total exclusiva más insulina, con restricción por vía oral en forma prolongada. Necesitó de asistencia psicológica y psiquiátrica durante la internación. La anatomía patológica mostró nesidioblastosis y lesión intraepitelial ductal de bajo grado. Luego de tres semanas de la cirugía, la paciente fue externada en tratamiento con insulina glargina $16 \mathrm{U}$, correcciones con insulina cristalina y enzimas pancreáticas.

En este caso, con síntomas graves de hipoglucemia y un diagnóstico muy poco frecuente de nesidioblastosis, fue necesario implementar un tratamiento agresivo con la secuela de diabetes insulino-requirente e insuficiencia pancreática exocrina.

\section{CONCLUSIONES}

La hipoglucemia hiperinsulinémica postprandial luego de la cirugía bariátrica es un cuadro más frecuente de lo que se sospechaba, que muchas veces pasa inadvertido, y se asocia con complicaciones desde leves hasta graves como convulsiones y coma. Es necesario tener un alto nivel de alerta para identificarla y de fundamental importancia identificar la causa de la hipoglucemia para orientar el tratamiento adecuado. En un alto porcentaje de casos el manejo nutricional puede prevenir los episodios de hipoglucemia; en los que no responden a los cambios de la dieta puede ser necesaria la medicación, y en algunos casos debe indicarse el tratamiento quirúrgico como último recurso.

\section{BIBLIOGRAFÍA}

1. Eisenberg D, Azagury D, Ghiassi S, et al. ASMBS. Position statement on postprandial hyperinsulinemic hypoglycemia after bariatric surgery. Surgery for Obesity and Related Diseases 2017; 13: 371-378.

2. Aasheim E, Frigstad SO, SøvikT, et al. Hyperinsulinemic hypoglycemia and liver cirrhosis presenting after duodenal switch: a case report. Surgery for Obesity and Related Diseases 2010; 6: 441-443.

3. Calabria AC, et al. Postoperative surveillance and detection of postprandial hypoglycemia after fundoplasty in children. J Pediatr 2011; 159 (4):597-601.

4. Lee CJ, Clark JM, Schweitzer M, et al. Prevalence of and risk factors for hypoglycemic symptoms after gastric bypass and sleeve gastrectomy. Obesity 2015; 23(5): 1079-84. 
5. Scavini M, Pontiroli AE, Folli F. Asymptomatic hyperinsulinemic hypoglycemia after gastricbanding. N Engl J Med 2005; 353:2822-2823.

6. Berkowitz D, Glassman S. Carbohydrate metabolism in the subtotal gastrectomy patient. Am J Gastroenterol 1966; 46:119-29.

7. Service GJ, Thompson GB, Service FJ, et al. Hyperinsulinemic hypoglycemia with nesidioblastosis after gastric bypass surgery. NEJM 2005; 353(3):249-54.

8. Kellogg TA, Bantle JP, Leslie DB, et al. Post gastric bypass hyperinsulinemic hypoglycemia syndrome: characterization and response to a modified diet. Surg Obes Relat Dis 2008; 4 (4):492-499.

9. Laurenius A, Werling M, Le Roux C, et al. More symptoms but similar blood glucose curve after oral carbohydrate provocation in patients with a history of hypoglycemia-like symptoms compared to asymptomatic patients after Roux-en-Y gastric bypass. Surg Obes Relat Dis 2014; 10:1047-1055.

10. Vezzosi D, Bennet A, Fauvel J, et al. Insulin, C-peptide and proinsulin for the biochemical diagnosis of hypo-glycemia related to endogenous hyperinsulinism. Eur J Endocrinol 2007; 157: 75-83.

11. Lev-Ran A, Anderson RW. The diagnosis of postprandial hypoglycemia. Diabetes 1981; 30:996 -999.

12. Hanaire $H$, Bertrand $M$, Guerci $B$, et al. High glycemic variability assessed by continuous glucose monitoring after surgical treatment of obesity by gastric bypass. Diabetes Technol Ther 2011; 13:625-630.

13. Halperin F, Patti ME, Skow M, et al. Continuous glucose monitoring for evaluation of glycemic excursions after gastric bypass. J Obes 2011. Doi:10.1155/2011/869536.

14. Abrahamsson N, Eden-Engstrom B, Sundbom M, et al. Hypoglycemia in everyday life after gastric bypass and duodenal switch. Eur J Endocrinol. 2015 Jul;173(1):91-100.

15. Kefurt R, Langer F, Schindler K, et al. Hypoglycemia after Rouxen- $Y$ gastric bypass: detection rates of continuous glucose monitoring (CGM) versus mixed meal test. Surg Obes Relat Dis 2015; 11:564-572.

16. Nielsen JB, Abild CB, Pedersen AM, et al. Continuous glucose monitoring after gastric bypass to evaluate the glucose variability after a low-carbohydrate diet and to determine hypoglycemia. Obes Surg 2016; 26(9): 2111-2118.

17. Malik S, Mitchell J, Steffen K, et al. Recognition and management of hyperinsulinemic hypoglycemia afterbariatric surgery. Review. Obes Res Clin Pract 2016; 10(1): 1-14.

18. Christfort- $Ø$ hrstrøm C, Worm D, Lindqvist-Hansen D. Postprandial hyperinsulinemic hypoglycemia after Roux-en-Y gastric bypass: an update. Surg Obes Relat Dis 2017; 13: 345-351.

19. Nannipieri M, Belligoli A, Guarino D, et al. Risk factors for spontaneously self-reported postprandial hypoglycemia after bariatric surgery. J Clin Endocrinol Metab 2016; 101 (10): 3600-3607.

20. Lee CJ, Wood GC, Lazo M, et al. Risk of post-gastric bypass surgery hypoglycemia in nondiabetic individuals: a single center experience. Obesity 2016; 24 (6): 1342-1348.

21. Mulla CM, Storino A, Yee EU, et al. Insulinoma after bariatric surgery: diagnostic dilemma and therapeutic approaches. Obes Surg 2016; 26:874-881.

22. Zagury L, Moreira RO, Guedes EP, Coutinho WF, Appolinario JC. Insulinoma misdiagnosed as dumping syndrome afterbariatric surgery. Obes Surg 2004; 14:120-123.
23. Kauhanen S, Seppänen M, Minn H, Gullichsen R, Salonen A, Alanen $\mathrm{K}$, et al. Fluorine-18-L-dihydroxyphenylalanine (18FDOPA) positron emission tomography as a tool to localize aninsulinoma or beta-cell hyperplasia in adult patients. J Clin Endocrinol Metab 2007; 92(4):1237-1244.

24. Rösch T, Lightdale CJ, Botet JF, et al. Localization of pancreatic tumors byendoscopic ultrasonography. N Engl J Med 1992; 326(26):1721-1726.

25. Doppman JL, Miller DL, Chang R, et al. Insulinomas: localization with selective intaarterial injection of calcium. Radiology 1991; 178(1):237-241.

26. Laferrere B, Reilly D, Arias S, et al. Differential metabolic impact of gastric bypass surgery versus dietary intervention in obese diabetic subjects despite identical weight loss. Sci Transl Med 2011; 3:80re2.

27. Service FJ, Natt N, Thompson GB, et al. Noninsulinoma pancreatogenous hypoglycemia: a novel syndrome of hyperinsulinemic hypoglycemia in adults independent of mutations in Kir6.2 and SUR1 genes. J Clin Endocrinol Metab 1999; 84(5): 1582-1589.

28. Marsk R, Jonas E, Rasmussen F, Naslund E. Nationwide cohort study of post-gastric bypass hypoglycaemia including 5,040 patients undergoing surgery for obesity in 1986-2006 in Sweden. Diabetologia 2010; 53: 2307-11.

29. Lee CJ, Clark JM, Schweitzer M, et al. Prevalence of and risk factors for hypoglycemic symptoms after gastric bypass and sleeve gastrectomy. Obesity 2015; 23(5): 1079-1084.

30. Harness JK, Geelhoed GW, Thompson NW, et al. Nesidioblastosis in adults. Arch Surg 1981; 116: 575-80.

31. Cui Y, Elahi D, Andersen DK. Advances in the etiology and management of hyperinsulinemic hypoglycemia after Roux-en-Y gastric bypass. J Gastrointest Surg 2011; 15: 1879-88.

32. Roth DA, Meade RC. Hyperinsulinism hypoglycemia in the postgastrectomy patient. Diabetes 1965; 14: 526-528.

33. Perfetti R, Zhou J, Doyle ME, et al. Glucagon-likepeptide-1 induces cell proliferation and pancreatic-duodenum homeobox-1 expression and increases endocrine cell mass in the pancreas of old, glucose-intolerant rats. Endocrinology 2000; 141:46004605.

34. Patti ME, Li P, Goldfine AB. Insulin response to oral stimuli and glucose effectiveness increased in neuro-glycopenia following gastric bypass. Obesity 2015; 23(4):798-807.

35. Meier JJ, Butler AE, Galasso R et al. Hyperinsulinemic hypoglycemia after gastric bypas surgery is not accompanied by islet cell hyperplasia or increased beta-cell turnover. Diabetes Care 2006; 29:1554-9.

36. Goldfine AB, Mun EC, Devine E, et al. Patients with neuroglycopenia after gastric bypass surgery have exaggerated incretin and insulin secretory responses to a mixed meal. J Clin Endocrinol Metab 2007; 92: 4678-4685.

37. Buchwald H, Estok R, Fahrbach $\mathrm{K}$, et al. Weight and type 2 diabetes after bariatric surgery: systematic review and meta analysis. Am J Med 2009; 122:248-256.

38. Beckman LM, Beckman TR, Sibley SD, et al Changes in gastrointestinal hormones and leptin after Roux-en-Y gastric bypass surgery. J Parenter Enteral Nutr 2011; 35: 169-180.

39. Rariy CM, Rometo D, Korytkowski M. Post-gastric bypass hypoglycemia. Curr Diab Rep. 2016; 16(2):19.

40. Salehi M, Gastaldelli A, D'Alessio DA. Blockade of glucagonlike peptide-1 receptor corrects postprandial hypoglycemia aftergastric bypass. Gastroenterology 2014; 146(3):669-680. 
41. Michaels A, Mehaffey H, French B, et al. Hypoglycemia following bariatric surgery: our 31-year experience. Obes Surg 2017; 27(12): 3118-3123.

42. Bantle A, Wang Q. Bantle J. Post gastric bypass hyperinsulinemic hypoglycemia fructose is a carbohydrate which can be safely consumed. J Clin Endocrinol Metab 2015; 100:3097-3102.

43. Millstein R, Lawler H. Hypoglycemia after gastric bypass: an emerging complication. Cleve Clin J Med 2017; 84 (4): 319-328.

44. Valderas JP, Ahuad J, Rubio L, et al. Acarbose improves hypoglycaemia following gastric by pass surgery without increasing glucagon like peptide 1 levels. Obes Surg 2012; 22:582-586.

45. Ritz $P$, Vaurs $C$, Bertrand $M$, et al. Usefulness of acarbose and dietary modifications to limit glycemic variability following Roux en $Y$ gastric bypass as assessed by continuous glucose monitoring. Diabetes Technology \&Therapeutics 2012; 14:736-740

46. Tack J, Arts J, Caenepeel P, et al. Pathophysiology, diagnosis and management of postoperative dumping syndrome. Nat Rev Gastroenterol Hepatol 2009; 6:583-590.

47. Craig C, Liu L, Nguyen T, et al. Efficacy and pharmacokinetics of subcutaneous exendin (9-39) in patients with post-bariatric hypoglycaemia. Diabetes Obes Metab. 2018; 20:352-361.

48. Patti ME, Goldfine A. Hypoglycemia after gastric bypass: the dark side of GLP-1. Gastroenterology 2014; 146 (3): 605-608.

49. Abrahamsson N, Engstrom BE, Sundbom M, et al. GLP1 analogs as treatment of postprandial hypoglycemia following gastric bypass surgery: a potential new indication? Eur J Endocrinol 2013; 169(6): 885-889.

50. Jammah A. Endocrine and metabolic complications after bariatric surgery. Review article. Saudi J Gastroenterol 2015; 21:269-277.

51. Bernard B, Kline GA, Service FJ. Hypoglycemia following upper gastrointestinal surgery: case report and review ofthe literature. BMC Gastroenterol 2010; 10:77.

52. Ceppa EP, Ceppa DP, Omotosho PA, et al. Algoritm to diagnose etiology of hypoglycemia after Roux-en-Y gastric bypass for morbid obesity: case series and review of the literature. Surg Obes Relat Dis 2012; 8:641-647.
53. MalaT. Postprandial hyperinsulinemic hypoglycemia after gastric bypass surgical treatment. Review article. Surgery for Obesity and Related Diseases 2014; 10:1220-1225.

54. Z'graggen K, Guweidhi A, Steffen R, et al. Severe recurrent hypoglycemia after gastric bypass surgery. Obes Surg 2008; 18: 981-988.

55. Campos G, Ziemelis M, Paparodis R, et al. Laparoscopic reversal of Roux-en-Y gastric bypass:Technique and utility for treatment of endocrine complications. Surg Obes Relat Dis 2014; 10: 36-43.

56. Shoar $S$, Nguyen $T$, Ona $M$, et al. Roux-en- $Y$ gastric bypass reversal: a systematic review. Surg Obes Relat Dis 2016; 12: 1366-1372.

57. Zorron R, Branco A, Sampaio J, et al. One-anastomosis jejunal interposition with gastric remnant resection (Branco-Zorron Switch) for severe recurrent hyperinsulinemic hypoglycemia after gastric bypass for morbid obesity. Obes Surg 2017; 27:990-996.

58. Mc Laughlin T, Peck M, Holst J, et al. Reversible hyperinsulinemic hypoglycemia after gastric bypass: a consequence of altered nutrient delivery. J Clin Endocrinol Metab 2010; 95:1851-1855.

59. Arora I, Patti ME. Can reversal of RYGB also reverse hypoglycemia? Molecular Metabolism 2018; 9: 1-3.

60. Mathavan VK, Arregui M, Davis C, et al. Management of postgastric bypass noninsulinoma pancreatogenous hypoglycemia. Surg Endosc 2010; 24: 2547-2555.

61. Singh E, Vella A. Hypoglycemia after gastric bypass surgery. Diabetes Spectr 2012; 25: 217-21.

62. Kirwan J, Aminian A, Kashyap S, et al. Bariatric surgery in obese patients with type 1 diabetes. Diabetes Care 2016; 39: 941-948.

63. Faucher $\mathrm{P}$, Poitou $\mathrm{C}$, Carette $\mathrm{C}$, et al. Bariatric surgery in obese patients with type 1 diabetes: effets on weigth loss and metabolic control. Obes Surg 2016; 26: (10) 2370-2378.

64. Mahawar K, De Alwis N, CarrW, et al. Bariatric surgery in type 1 diabetes mellitus: a systematic review. Obes Surg 2016; (26): 196-204.

65. Chow A, Switzer N, Dang J, et al. A systematic review and meta-analisis of outcomes for type 1 diabetes after bariatric surgery. Journal of Obesity 2016. 